\title{
Lumières sur la guerre : ambivalence des symboles dans quatre récits de la Grande Guerre
}

\author{
Flavie FOUCHARD \\ Universidad de Sevilla \\ flaviefouchard@us.es \\ ORCID: 0000-0002-9895-8203
}

\section{Resumen}

En este artículo procedemos a una lectura atenta de algunos fragmentos de Gaspard de René Benjamin, Le Feu de Henri Barbusse, Les Croix de bois de Roland Dorgelès et Clavel Soldat de Léon Werth para analizar la manera en que sus autores tratan el motivo de la luz. Nos permite acercarnos a estas obras que nacen de la experiencia, centrándonos en las distintas interpretaciones del conflicto que construyen, a partir de una estética que va más allá del realismo y que utiliza el símbolo de la luz para cuestionar los símbolos y las representaciones tradicionales de la guerra, pero que también nos permiten atribuir diversos sentidos, a veces ambiguos, a una experiencia inédita de la guerra.

Palabras clave: Representación. Símbolo. Naturaleza. Primera guerra mundial.

\section{Résumé}

Dans cet article nous procédons à une lecture attentive des notations de lumière dans certains fragments de Gaspard de René Benjamin, Le Feu de Henri Barbusse, Les Croix de bois de Roland Dorgelès et Clavel Soldat de Léon Werth. Cette analyse nous permet d'aborder sous l'angle de l'interprétation du conflit qu'elles proposent ces œuvres qui prennent pour point de départ l'expérience mais ne se réduisent pas à l'écriture réaliste. En effet, la symbolique de la lumière leur permet de remettre en question les représentations traditionnelles de la guerre, mais aussi de donner leur propre interprétation, parfois ambiguë, de cette expérience guerrière radicalement nouvelle.

Mots clé : Représentation. Symbole. Nature. Première Guerre Mondiale.

\section{Abstract}

In this paper, we conduct a close reading of some fragments of Gaspard from René Benjamin, Le Feu from Henri Barbusse, Les Croix de bois from Roland Dorgelès and Clavel Soldat from Léon Werth to study the motif of light in their self-experience-based narration. We focus on light's symbolism that these non-exclusively realistic texts use to both express

\footnotetext{
* Artículo recibido el 11/11/2019, aceptado el 7/02/2020.
} 
critics on war's traditional representation and give an own interpretation, sometimes ambiguous, of a whole new war experience.

Keywords: Representation. Symbol. Nature. First World War.

\section{Introduction}

Pour de nombreux soldats de la Grande Guerre, dont les écrivains que nous allons évoquer, la nature est le cadre principal de la guerre. La campagne du Nord ou de l'Est de la France, dans les récits qui prennent pour ancrage le front sur le territoire français, occupe donc une place primordiale et recouvre par là-même de nombreuses significations au sein de textes dont les orientations sont, elles aussi, variées, comme l'explique Pierre Schoentjes (2011 : 123) : «La guerre de 14-18, première guerre industrielle et où la mécanisation a joué un rôle fondamental, illustre des rapports complexes dans la mesure où cette guerre "moderne" a eu pour cadre essentiel un univers rural et champêtre ». Ce critique propose une "typologie générale » des descriptions de la nature dans ces textes qui vise à mieux comprendre les différents rapports entre guerre et nature, et montre que ces relations ne se réduisent en aucun cas à une opposition stricte entre les deux termes (Schoentjes, 2011 : 125). Il dégage ainsi cinq types d'images de la nature qui s'inscrivent dans le prolongement d'une tradition bien ancrée : "la nature d'Arcadie, la nature de robinsonnade, la nature champêtre, la nature primitive et la nature apocalyptique » et oscillent entre deux pôles, celui de la nature idyllique, rappelant l'âge d'or d'avant le conflit ou se constituant en décor propice à un bref moment de répit pour le guerrier ; et celui de l'image du conflit représenté comme un enfer sur terre.

À partir des perspectives ouvertes par cette étude générale, nous avons souhaité étudier un élément de détail de la description, qui l'anime et qui participe du pouvoir symbolique de celle-ci : la lumière. Tout comme la relation de l'homme avec la nature, la portée symbolique de la lumière se voit affectée par le bouleversement qu'introduit la guerre dans les représentations traditionnelles de l'héroïsme et du combat. Tout comme le paysage, elle symbolise un large spectre de valeurs, concepts et réalités humaines : elle est traditionnellement associée, dans notre culture, à la connaissance, la gloire, l'héroïsme, la victoire, le sens, la clarté, le progrès ou encore le renouveau annoncé par l'aube. Elle participe alors des anciens stéréotypes sur la guerre, véhiculés avant et pendant le conflit, et qui circulent dans nos œuvres. La lumière contribue également à la création de nouvelles images qui fonctionnent, elles aussi, comme des clichés, dans des textes qui, pour mieux tenter de la représenter mettent au cour de leur projet littéraire "l'expérience sensorielle de la guerre » et 
transforment tout en «symbole $»^{1}$ (Beaupré, $2006: 158$ et 192).

L'étude du motif de la lumière va alors nous permettre de mettre en évidence les marques des tensions à l'œuvre dans chaque texte : entre une volonté de représenter la guerre dans sa réalité, de donner du sens à cette expérience et la difficulté à lui en trouver un acceptable (au regard de la morale, de la religion, de l'éthique, des idéaux républicains ${ }^{2}$ ou d'une idéologie politique, selon les textes). Nous allons nous pencher sur certains passages de Gaspard (1915) de René Benjamin, du Feu d'Henri Barbusse (1916), des Croix de bois (1919) de Roland Dorgelès et de Clavel Soldat de Léon Werth (1919), en cherchant à montrer comment les auteurs retravaillent les images de la tradition à leur disposition pour rendre compte d'une expérience qu'ils ont à la fois perçue comme extraordinaire et universelle. Nous nous inscrirons dans la lignée de Michèle Touret (2001 : 219) quand elle explique que cette situation a produit "une littérature où la "défamiliarisation" était le fait de la situation et non celui des formes qui, elles, effaçaient au contraire tout obstacle à la saisie immédiate et à l'entente des lecteurs". Il ne s'agit pas de faire des techniques employées dans ces textes des moyens représentatifs de tous les récits écrits par des écrivains combattants, mais de proposer une lecture approfondie de textes "classiques" à partir d'éléments de détails révélateurs. Nous examinerons les différents récits dans une perspective qui tentera de tenir compte des différences chronologiques (date d'écriture et de publication en relation avec le conflit) pour mieux appréhender les différentes occurrences des images ${ }^{3}$. Avant de commencer notre parcours, nous ferons un point sur les deux grands types de lumière qui se retrouvent dans ces récits : la lumière naturelle et celle qui émane des machines et de la guerre moderne. Ce qui nous permettra de contextualiser le débat esthétique et moral au cœur de la représentation littéraire de la guerre.

\section{L'" aurore boréale du champ de bataille »(Duhamel, $1967: 31)$}

Dans les récits que nous allons analyser, nous retrouvons deux types d'éclairages : l'un émane de la nature, l'autre de la guerre moderne et de ses instruments ou des situations qui lui sont propres. Nous allons d'abord brièvement évoquer la seconde, plus citée et étudiée que la première, puisque les notations des lumières de

\footnotetext{
${ }^{1}$ Dans la continuité de son travail dans Fictions de la Grande Guerre (2009), Pierre Schoentjes (2011: 123) traite les images de la nature comme " lieu de cristallisation d'enjeux thématiques majeurs ".

${ }^{2}$ La représentation des soldats en électeurs est fréquente dans Gaspard, comme pour mieux interroger ce que la République attend de ses citoyens (Fouchard, 2017 : 146-147).

${ }^{3}$ Nicolas Beaupré (2013 : 171) explique qu'au sein même de la guerre comme événement, il faut distinguer plusieurs périodes en ce qui concerne les récits qui en témoignent : «Les horizons d'attente de 1914 (où domine la croyance en une guerre courte, voire très courte) ne sont assurément pas les mêmes qu'en 1916 pendant la bataille de Verdun ou qu'en 1917 après l'échec de l'offensive du Chemin des Dames ».
} 
la guerre moderne ont constitué l'un des clichés constitutifs de sa représentation. Cela nous permettra de revenir sur les difficultés que le choix d'une posture esthétique a posées à tous les auteurs ayant voulu témoigner de "leur" guerre car, comme l'explique de nouveau Pierre Schoentjes (2009: 44), "faire le récit de la guerre ne va pas sans problèmes".

La lumière qui illumine le front lors, notamment, des bombardements, est présente dans de nombreux poèmes, romans, récits et articles ayant pris pour sujet la première guerre mondiale. Nous la trouvons dans un texte de Colette daté de décembre-janvier 1915, "À Verdun ", dans lequel l'auteure commente son voyage incognito vers Verdun, qui n'est pas encore synonyme d'hécatombe, pour retrouver son mari, officier cantonné dans cette ville: "Heures longues? peut-être, à cause de l'impatience d'arriver, mais heures remplies, inquiètes, illuminées par la lueur boréale d'une canonnade incessante, une lueur rose qui halète au ras de l'horizon, au nordest " (Colette, 1984 : 490). C'est une image précoce, elle se généralise rapidement et devient même un stéréotype. Mais, elle possède toujours également pour objectif de faire voir la réalité de la guerre aux lecteurs qui ne connaissent pas le front, ressource largement utilisée par de nombreux auteurs, comme l'explique Laurence Campa (2010 : 33) : "Esthétiser la guerre ou tout autre sujet, c'est la rendre sensible au lecteur, par tous les moyens, et plus précisément produire des sensations. La poétiser, c'est en faire un matériau poétique ". Les descriptions de cette lumière, étonnement belle en regard de la destruction qu'elle annonce, est au cœur d'un dilemme plus large auquel ont été confrontés les écrivains qui voulaient témoigner littérairement de la guerre. En effet, comme l'explique Michèle Touret, cela constituait un véritable " [d]éfi à la littérature " car il s'agissait de choisir entre deux postures antagoniques, qui avaient nécessairement, au-delà des parti-pris esthétiques, des implications morales et des conséquences sociales : " abandonner la voie créatrice, peut-être indécente et impuissante, et témoigner en renonçant aux ressources habituelles de la littérature ; ou ne pas y renoncer mais courir le risque d'une création qui trahira ses objectifs et pourra rendre la guerre belle et aimable...» (Touret, 2001 : 218). Cette voie autorise la création d'images qui associent les lueurs de la guerre à des comparants naturels comme dans ce poème d'Apollinaire "Carte postale ", cité et commenté par Laurence Campa lors de son analyse de la réception des textes de guerre du poète :

Je t'écris de dessous la tente

Tandis que meurt ce jour d'été

Où floraison éblouissante

Dans le ciel à peine bleuté

Une canonnade éclatante

Se fane avant d'avoir été (Campa, 2001 : 297)

${ }^{4}$ Tiré de « La tête étoilée » dans Calligrammes. 
Elle remarque que, pour appréhender une expérience aussi neuve, le poète puise ici dans le répertoire traditionnel de la nature, "floraison, ciel à peine bleuté, se fane ", tout en le réinvestissant dans de nouvelles circonstances : " une canonnade éclatante ", ce qui confère au poème un mélange de proximité et d'étrangeté. Cette technique rend également patent le scandale que peut représenter la comparaison entre le cycle naturel, admiré par la tradition, synonyme de beauté grâce au terme "floraison ", avec les instruments de la guerre moderne. Seuls les termes " meurt " et "se fane " semblent mettre à distance cette représentation "de carte postale ", dont nous ne pouvons éviter de penser qu'elle suggère une certaine ironie.

Dans certains récits, les auteurs se veulent parfois plus explicites quant aux significations de ces lumières. Dans ce passage des Croix de bois c'est le paysage urbain qui sert de comparant ("long boulevard de clarté »; " enseignes lumineuses »), ainsi que les célébrations de la fête nationale pendant les soirs d'été :

Dans un bourdonnement assourdi de voix étouffées, de cliquetis et de pas fourbus, la compagnie entra dans le village noyé d'ombre. Pas bien loin, les fusées barraient la nuit d'un long boulevard de clarté, et, par instants, cela s'égayait de lueurs rouges ou vertes, vite éteintes, pareilles à des enseignes lumineuses.

Ce ciel de guerre fait penser à une nuit populaire de 14 Juillet. Rien de tragique. Seul, le vaste silence (Dorgelès, 2013 : 34).

La comparaison avec le ciel de "nuit populaire de 14 Juillet » vise la vision édulcorée et patriotique de l'événement, depuis l'extérieur ${ }^{5}$. Nous pouvons penser qu'elle renvoie à un certain symbolisme attaché à l'événement, notamment au début du conflit. Les deux phrases nominales suivantes contrastent toutefois fortement avec cette imagerie, sans pour autant basculer vers le pôle du sensationnalisme ("Rien de tragique "). À quoi attribuer ici "le silence " ? À une notation réaliste, au manque de sens de l'événement, à l'indifférence qu'il provoque depuis la distance, à la sensation des soldats au plus profond de la nuit? Nous pouvons trouver une réponse dans la perception de Demachy, qui vient d'arriver sur le front, assumée par le narrateur omniscient :

Les yeux tournés vers les premières lignes, il cherchait cependant à voir les fusées, entre deux murs. C'était pour lui une déception, cette première vision de la guerre. Il aurait voulu être ému, éprouver quelque chose, et il regardait obstinément vers les tranchées, pour se donner une émotion, pour frissonner un

\footnotetext{
${ }^{5}$ Quelques pages plus loin, les camarades du narrateur regardent toutefois un bombardement avec « un cri d'admiration " et "une clameur ravie de feu d'artifice» (Dorgelès, 2013: 40). Dorgelès (2013: 118) file la métaphore tout au long du texte. Ainsi, retrouvons-nous cette mention au feu d'artifice lors de l'épisode du « mont Calvaire ».
} 
peu. [...] Il ne ressentait rien, qu'un peu de surprise. Cela lui semblait tout drôle et déplacé, cette féérie électrique au milieu des champs muets. [...] Même ce village dévasté ne le troublait pas : cela ressemblait trop à un décor. C'était trop ce qu'on pouvait imaginer (Dorgelès, $2013: 35$ ).

Celui qui s'approche des lueurs de la guerre pour la première fois, désireux pourtant de les connaître, éprouve une déception envers ce qui ressemble à un spectacle au milieu du silence : "féérie, décor ". L'expérience est en effet teintée de ses propres attentes, fabriquées au préalable par son "imagination », sûrement aidée par les représentations circulant à l'arrière. L'indifférence de la nature et de la nuit à l'agitation humaine n'évoque aucun pathétisme, sinon un sentiment d'irréalité : " cette nuit, ce grand silence, ce n'était pas la guerre... " Nous touchons ici à la difficulté de représenter la réalité guerrière pour ceux qui ne la vivent pas et ne la connaissent pas.

Alors, certains auteurs font le choix de traduire ce que signifient les dessins et lueurs que tracent dans le ciel les «canonnades". Ainsi, Duhamel, s'attache lui aussi à l'" aurore boréale du champ de bataille " dans "Sur la Somme" (Civilisation), en n'oubliant pas de faire intervenir le responsable humain de ce cataclysme :

Quel orage humain! Quelle explosion de haine et de destruction! On eût dit qu'avec des millions d'étincelles une troupe de géants forgeaient l'horizon de la terre en frappant dessus à coups redoublés. Faite d'une infinité de lueurs furtives, une immense lueur continue vivait, palpitait, bondissait, éblouissant le paysage et la nue. Des gerbes irisées fusaient en plein ciel, comme le marteau-pilon en exprime de la fonte incandescente.

Pour moi qui sortais de la tranchée, tous ces artifices signifiaient quelque chose, des recommandations, des ordres, des appels désespérés, des signaux d'égorgement, et je commentais ce brasier comme s'il eût exprimé en toutes lettres la fureur et la détresse des combattants (Duhamel, $1967: 31$ ).

Dans ce passage, Duhamel commence par évoquer la responsabilité humaine dans ce déchaînement. Mais, comme si la haine et la destruction étaient incompatibles avec les images lumineuses: "étincelles, lueurs, immense lueur continue, éblouissant, gerbes irisées, incandescente " ou même vitales : "furtives, vivait, palpitait, bondissant "; Duhamel tente de faire revenir le lecteur à la vraie signification de ce spectacle. Il interprète "ce brasier " pour ceux qui ne sortaient pas de la tranchée, comme lui, et attache à son texte la fonction du "commentaire " qui permet à l'écriture réaliste de dépasser le simple objectif de "rendre sensible " la guerre au lecteur. Dans cette entreprise, la lumière naturelle qui éclaire les scènes de ces récits joue un rôle symbolique fort. 
D’une part, elle a été contaminée par les nouveaux instruments de la guerre moderne, plus guère absents des paysages, comme le montre ce passage des Croix de bois, lorsque la compagnie du narrateur arrive dans la tranchée, à l'aube, pour la relève :

Un joli soleil pâle de la Saint-Martin. Sur le ciel d'un bleu tendre, les nuages étaient pareils à des flocons de shrapnells. Un émouchet et un corbeau se poursuivaient à coups de bec, sauvagement. On entendait une alouette, qui bougeait à peine. C'était dimanche (Dorgelès, 2013 : 38).

La beauté de la matinée de ce dimanche de fête, faiblement ensoleillée, est rapidement réinsérée dans le temps guerrier grâce au comparant associé aux nuages. La brutalité envahit alors le paysage à travers la scène qui fait s'affronter, symboliquement, un rapace et un charognard, ce dernier habitué des champs de bataille. Nous voyons ici que les éléments de la guerre moderne envahissent logiquement les descriptions de la nature et lui donnent, à cette occasion, une coloration inquiétante. D'autant plus que la lumière solaire, éclatante, a traditionnellement symbolisé la victoire et la gloire et a donc, à ce titre, fait l'objet d'un travail de mise à distance important de la part de nos auteurs.

\section{Les illusions de la victoire ensoleillée dans Gaspard}

Dès Gaspard, roman écrit en 1914 et qui met en scène son personnage de la déclaration de la guerre à l'immobilisation du conflit dans les tranchées, les effets de lumière confirment, dans un premier temps, l'association qui se fait traditionnellement entre une victoire pressentie comme rapide et facile et le caractère étincelant. Or, dans ce roman généralement considéré comme un modèle du genre patriotique, le récit mène Gaspard à perdre ses illusions sur la guerre, ce qui s'accompagne d'un changement d'éclairage, déjà présent dans les errements prémonitoires du temps au cours de la "grande semaine d'août 1914, où chaque ville de chaque province offrit un régiment à la France " (Benjamin, 1998 : 19). Ainsi, le soir du 2 août, premier jour de Mobilisation Générale, la lumière donne une tonalité "heureu[se] " à la fin de la journée : «la soirée était belle. Le soleil baissant, les toits devenaient roses. Un rayonnement heureux caressait les maisons. Le bleu du ciel, en s'allégeant, donnait des ailes à la soirée ; et il y avait des promesses dans la tiédeur de l'air " (Benjamin, 1998 : 21). Mais le lendemain, le temps a changé - «Le beau temps de la veille s'était corsé et il faisait un soleil vigoureux qui coloriait de petits nuages ronds, sans poésie " (Benjamin, $1998: 22$ ) -, au point d'entraîner un orage :

Mais dans la nuit le vent changea brusquement, et une averse tomba drue sur la ville. Elle s'éveilla pourtant sous le ciel bleu. Il ne dura pas. De nouveau, toute une bande de petites nuées rapides s'en vinrent crever en trombes rageuses, dont l'eau séchait, sitôt tombée. [...] On ne savait si le temps riait ou pleu- 
rait. Matinée nerveuse, de colères et de rayonnements (Benja$\min , 1998: 22-23)$.

Les personnifications, dans ces deux exemples, (" heureux", "coloriait ", " riait ", " pleurait ") sont caractéristiques de la fonction symbolique que revêt la description du temps et de la nature, et par extension des notations de lumière. L'effet de réel $^{6}$ n'est pas le seul but de ce commentaire qui annonce déjà des perturbations : dans les plans tout tracés sur la guerre mais aussi dans l'ordre symbolique.

Avant de partir sur le front, encore à la caserne, Gaspard connaît le « réveil en fanfare " du soleil :

Heureusement, les nuits d'août sont courtes, et les idées noires des hommes ne résistent guère à un lever de soleil éclatant, qui met d'abord le ciel en feu, puis qui lance à la terre tous ses rayons ensemble, qui s'impose, qui ne laisse pas libre une seule fenêtre, qui vient chercher le dormeur sur sa paille, l'éblouit paupières baissées, lui sonne un réveil en fanfare, illuminant toutes ses idées [...] (Benjamin, $1998: 36$ ).

Ce lever du jour lui fait vite oublier l'épisode de la veille, au cours duquel un sergent-major avait demandé aux recrues l'adresse de leur famille "en cas de décès ", cynisme qui avait momentanément révolté Gaspard. Ce matin-là, il devient semblable à ses compagnons "qui prenaient confiance dans ce bain de jour bienveillant et prometteur" (Benjamin, 1998 : 37). En ce début de roman, l'idée d'une victoire facile circule dans les rues de la ville d'Alençon, où se trouve cantonné le régiment de Gaspard, avant son départ. Plusieurs notations montrent que cette idée vient de la presse, des communiqués officiels et de la rumeur publique. Gaspard ainsi que les autres personnages se l'approprient et paradent dans la ville en la faisant circuler à grand renfort de gestes et de fanfaronnades.

Le règne de l'aube se maintient malgré les premières offensives et les premières troupes de blessés qui passent en débandade devant Gaspard et ses compagnons d'armes :

Le jour se levait; l'horizon devenait gris ; les hommes, entre eux, commençaient à distinguer leurs visages livides. Et soudain, la campagne s'éclairant, le régiment s'aperçu qu'il n'était plus seul dans cette marche en avant. [...] Avec l'aube, plus de blessés. Le soleil montait, face aux hommes. On marchait vers l'Est, droit sur lui [...]. On était dégagé. On respirait. On se sentait une troupe neuve et forte. On s'en allait vers le Destin,

\footnotetext{
${ }^{6}$ Nous retrouvons cette description du temps estival par exemple chez Colette dans le texte court « La nouvelle » inclus dans Les Heures longues: " Quatre heures, un beau jour voilé d'été marin, les remparts dorés de la vieille ville debout devant une mer verte sur la plage, bleue à l'horizon » (Colette, 1984: 478).
} 
et il paraissait devoir être la victoire plutôt que le massacre (Benjamin, 1998 : 97-98).

De nouveau, le soleil instille sa force aux soldats, mais c'est ici encore un prélude car ceux-ci n'ont pas encore vraiment fait l'expérience directe du combat. En raison de leur manque d'expérience et de la puissance ascendante du symbolisme de l'aube ("le soleil montait»), les hommes trouvent encore que le temps accompagne leur désir de gloire. Ce n'est qu'après la première attaque que la lumière devient, pour Gaspard, le rappel de l'extrême violence des combats qu'il a vécus et nimbe sa réminiscence d'une « journée fantastique »:

Il faisait nuit, une nuit de lune pour contes de fées, et pourtant il avait encore plein la tête, plein les yeux, la lumière violente de la journée fantastique qu'il venait de vivre, et dont il lui restait un enchevêtrement d'images atroces, avec une impression durable de vacarme infernal (Benjamin, 1998 : 127).

L'astre qui accompagne son retour d'attaque, lors de laquelle il a perdu son ami Burette et s'est vu infliger sa première blessure, est maintenant la lune. Tirée des " contes de fées ", elle ne parvient pas à faire oublier la "lumière violente de la journée fantastique ". Ces deux caractérisations des notations lumineuses font allusion au merveilleux et à l'étrangeté de l'expérience vécue. L'expérience semble se déréaliser via la lumière qui s'en dégage. Ainsi, après avoir eu recours aux clichés sur l'aube glorieuse, tout aussi éloignés de la réalité mais assumés comme vrais par le personnage, au début de son aventure; le narrateur éclaire cette scène et la réminiscence de l'antérieure de lueurs surnaturelles. Nous pouvons alors nous interroger sur le symbolisme de la lumière que projette la lune sur le récit, difficile à dégager, notamment en raison de son rôle dans la suite du passage.

En effet, la lune est omniprésente dans cette scène où elle sert d'abord à éclairer de manière singulière le trajet de Gaspard vers l'arrière, en compagnie des autres blessés : "Têtes d'épopée, soudain toutes blêmes, ensuite dans l'ombre, à la fois terribles et comiques sous des képis drôlement juchés, qui donnaient des airs de fantoches à ces humains si pitoyables » (Benjamin, 1998 : 131). Elle révèle la nature de la scène qui est en train de se jouer pour les hommes qui ont survécu au déchaînement de la violence : le passage de l'héroïsme au pathétisme et au comique. Par ailleurs, l'insistance du narrateur à mentionner l'éclairage continu de la lune, ce soir-là "si brillante ", lui permet de représenter de manière paradoxale la scène de l'arrivée dans l'église qui abritera les soldats, avant leur rapatriement vers l'arrière.

La connotation de rêverie lyrique ou poétique que possède cet astre ne se transmet ni au personnage principal, qui poursuit ses aventures de manière tout aussi gaillarde; ni aux autres soldats : "La lune, si brillante, devait paraître odieuse à ces malheureux. Ils avaient fui sa clarté pâle " (Benjamin, 1998 : 131); ni à la scène. Une fois entré dans l'église, Gaspard pousse en effet une statue du Christ pour s'installer 
sur une botte de paille, à côté d'un autre blessé, et attendre. L'éclairage de la lune les surprend mais aucune transcendance ne se dégage, bien au contraire : «Il s'étendit de l'autre côté, dans le clair de lune qui donnait sur le Christ et sur eux ; ils avaient l'air des deux larrons ; et ils se mirent à parler et à se plaindre par-dessus la croix » (Benjamin, 1998 : 132). Malgré le commentaire relativement long du narrateur, à la page suivante, sur la portée de cette scène visant à évoquer « la misère humaine, qui interroge et qui supplie ", grâce à sa représentation à l'intérieur d'une église à moitié détruite, la construction du stéréotype du martyr, fréquemment utilisé dans la représentation du soldat ${ }^{7}$, a du mal à fonctionner auprès du lecteur. En effet, la trivialité de Gaspard, et l'apparition soudaine du jour - « La lune disparut. Le jour commença de naître " (Benjamin, 1998 : 133) - coupent court à toute envolée lyrique et marquent le retour définitif à la réalité : "C'était, dans la première clarté lugubre du jour, une mêlée à décourager les meilleurs" (Benjamin, 1998 : 134). Le soleil et les engouements qu'il suscitait ont disparu avec l'expérience du front, la lueur de la lune ne semble pas être à même de lui redonner son brio perdu.

Au fil du texte de Benjamin, qui suit les premiers moments du conflit, de la guerre de mouvement jusqu'à l'établissement des tranchées, le narrateur opère de manière assez schématique mais aussi parfois subtile, un changement d'éclairage qui fait perdre de son éclat à la gloire et transforme les paysages guerriers. Quand, après six mois de convalescence, Gaspard revient sur le front au "bois de la Tuerie" ", il découvre les tranchées qu'il n'a pas connues lors de sa première campagne. Ce nouveau paysage ne possède plus qu'une lumière tamisée. Il est "brumeux " et se compose de "pluie ", de «brume», de "brouillard " et de "grisaille de brume " (Benjamin, 1998 : 221-222). Dorgelès reprendra également ces notations pour amalgamer aux notations réalistes sur la temporalité de l'action les sensations d'épuisement des soldats. Il décrit par exemple " un brouillard de fatigue et de pluie » qui enveloppe les soldats de sa compagnie sur le chemin du cantonnement (Dorgelès, 2013 : 51).

Barbusse, lui, tirera un maximum d'effet de cet éclairage dans Le Feu, notamment dans les contrastes que cette nouvelle lumière permet de construire. Ainsi, dans le chapitre "Le Chien ", alors que les soldats de l'escouade à laquelle appartient le narrateur sont en cantonnement dans un village, le poilu Fouillade se lave à grandes eaux au milieu du village, par un "temps épouvantable» (Barbusse, 1965 : 177). Lorsqu'il se met enfin à l'abri, Fouillade est pris d'une rêverie qui l'entraîne sur le chemin de son Hérault natal : «Il revoit. C'est un de ces moments où le pays dont on est séparé prend, dans le lointain, des douceurs de créature " (Barbusse, 1965 : 184). Après avoir placé la scène du bain sous une lumière des plus tristes, "le paysage de ce matin-là était jaune sale, le ciel tout noir - couvert d'ardoises ", le narrateur

\footnotetext{
${ }^{7}$ Sur cette figure, lire le point «Le Martyre " dans Écrire en guerre, écrire la guerre France, Allemagne 1914-1920 (Beaupré, 2006).
} 
insiste sur la beauté du paysage de l'enfance de Fouillade. Entre mer et montagne et, surtout, baigné par un soleil éblouissant qui donne ses couleurs aux repas de famille, " la lumineuse odeur d'ail de sa table lointaine " (Barbusse, 1965 : 186), et au souvenir de la rencontre avec sa femme : "La première fois, elle passait, luxueuse de soleil " (Barbusse, 1965 : 185). Bien sûr, le contraste appartient ici au répertoire des représentations stéréotypées de la nature qui oppose l'abondance et la joie se dégageant d'un paysage solaire, antérieur à la guerre, et la misère du soldat sous la pluie, loin de sa terre natale et en proie au bourdon. Mais l'intérêt de ce retour aux souvenirs lumineux de l'enfance ne réside pas seulement dans l'effet de pathétique que Barbusse peut en tirer.

À l'occasion de cette évocation, il revient sur cette époque où l'âge d'or de l'enfance coïncidait avec une conception erronée de la guerre, auréolée d'une gloire lumineuse :

Il jouait, sur la terre dorée et rousse, et même il jouait au soldat. L'ardeur de manier un sabre de bois animait ses joues rondes qui sont maintenant ravinées et comme cicatrisées... Il ouvre les yeux, regarde autour de lui, hoche la tête, et s'adonne au regret du temps où il avait un sentiment pur, exalté, ensoleillé de la guerre et de la gloire (Barbusse, 1965 : 184).

Le lexique de la lumière («dorée ») et de la vitalité («ardeur, animait»), accompagnent la caractérisation du sentiment nostalgique et permettent le retour à l'idéalisation passée de la guerre et de la gloire. La pureté de la vision de l'enfance ancrée dans le jeu ("sabre de bois»), fait place à la réalité présente ("ravinées et comme cicatrisées »). L'intense lumière du soleil sert donc à Barbusse à signaler deux brèches ouvertes par le conflit, qui ne cessent de s'agrandir au fil du récit : celle qui existe entre le temps de la guerre et celui du passé, présenté ici comme heureux ; mais aussi entre la conception passée de la guerre et l'expérience réelle de celle-ci.

\section{Pluie, brume, brouillard et aube blafarde : réalisme et temporalité du récit de guerre}

Dans le chapitre d'ouverture du Feu, le narrateur peint un tableau alpin où les "lueurs sinistres d'[un] orage " accompagnent l'annonce de la guerre et éclairent " une grande plaine livide» (Barbusse, 1965: 27). Au deuxième chapitre, qui commence in medias res, cet orage est transposé sur le front et l'aube se lève sur un paysage dévasté : "La terre ! Le désert commence à apparaître, immense et pleine d'eau, sous la longue désolation de l'aube" (Barbusse, 1965 : 28). À partir de ce moment, la pluie et "la lumière blafarde" s'installent durablement sur le décor du récit ${ }^{8}$.

${ }^{8}$ À ce sujet, Pierre Schoentjes (2011 : 134) signale que "malgré un titre qui promet "le feu", l'ouvrage de Barbusse créé un univers apocalyptique qui est d'abord fait d'eau. L'idée de déluge est constamment reprise dans ce roman qui multiplie les paysages sous la pluie, l'évocation des fondrières et des torrents 
Quelques pages plus loin, la lumière du jour ne parvient ni à percer, ni à donner de l'espoir aux hommes :

La journée s'avance. Un peu plus de lumière a filtré des brumes qui enveloppent la terre. Mais le temps est resté couvert, et voilà qu'il se résout en eau. [...] Le brouillard et les gouttes empâtent et ternissent tout [...]. Sur la terre, champ de mort, se juxtapose étroitement le champ de tristesse du ciel (Barbusse, $1965: 52)$.

L'expérience du temps de la guerre est ainsi rendue, notamment par les notations lumineuses qui accompagnent l'attente et la difficulté à occuper les journées sur le front : "Ce sera dur, aujourd'hui, de venir à bout de la journée, de se débarrasser de l'après-midi » (Barbusse, 1965 : 52). Dans des narrations qui s'effilochent ${ }^{9}$, pour mieux représenter une guerre où l'attente est omniprésente, "On attend toujours, dans l'état de guerre. On est devenus des machines à attendre " (Barbusse, 1965 : 44), la notation d'un effet de lumière, d'un changement d'éclairage, peut se présenter comme un événement qui signale le passage du temps. Dorgelès (2013 : 179) nous livre, à ce propos, ces pensées du narrateur : "Alors, on se rassied, le dos au mur, et on attend. Faire la guerre n'est plus que cela : attendre. Attendre la relève, attendre les lettres, attendre la soupe, attendre le jour, attendre la mort... Et tout cela arrive, à son heure : il suffit d'attendre... ". L'arrivée du jour est bien considérée comme un événement pareil à un autre.

Dans nos récits, il n'est pas rare que des chapitres ou parties de chapitre commencent par une notation temporelle, accompagnée par une notation lumineuse, pour situer l'action. Comme ici, au début de la dernière section du premier chapitre de Croix de bois, intitulé "Frères d'armes": "La nuit tombe vite, en novembre. Avec l'ombre, le froid était venu et là-bas, aux tranchées, la fusillade s'était éveillée, à l'heure des hiboux " (Dorgelès, $2013: 20$ ). Cette notation introduit la scène narrative du coucher des soldats. Celui-ci clôt la scène de présentation des personnages, organisée autour de l'arrivée de "trois soldats ahuris " (Dorgelès, 2013 : 12), fraîchement sortis de la caserne, au cantonnement des « anciens », dont fait partie le narrateur.

Ce type de description, par sa récurrence, nous signale une des spécificités de l'expérience guerrière des soldats du front, dont de nombreux auteurs et soldats, dans leurs écrits privés, ont tenté de rendre compte :

À partir du moment où les soldats s'enterrent, descendent dans le sol, s'installent dans une immobilité physique, le temps semble également comme se figer. La perspective de la fin de la guerre s'éloignant avec cette réalité stratégique nouvelle, les ho-

de boue ». Pour une étude du symbolisme de l'Apocalypse et du Déluge dans Le Feu, voir l'article de Sylvain Ledda "Requiem ou symphonie funèbre ? Notes sur la mort dans Le Feu " (2015).

${ }^{9}$ Pour Clavel Soldat, Jean Kaempfer parle de « déréliction anecdotique » (1998: 262). 
rizons d'attente, sans disparaître totalement, deviennent malgré tout flous, brumeux (Beaupré, 2013 : 176).

Ainsi, les effets de lumière tentent-ils, dans ce cas, de symboliser un nouveau sentiment face au passage du temps sur le front et à la monotonie des journées et de la "répétition cyclique et interminable des jours de guerre" (Beaupré, 2013 : 178). Expérience qui distingue le temps du front de celui de l'arrière en ce qu'elle semble à certains moments suspendre le temps dans un présent sans issue (Beaupré, 2013: 177). Comme l'exprime bien Dorgelès (2013 : 244), rétrospectivement, avec toute la distance qu'a mis la fin de la guerre avec son expérience, dans le dernier chapitre de son récit : "On parlait de sa vie comme d'une chose morte, la certitude de ne plus revenir nous en séparait comme une mer sans limites, et l'espoir même semblait s'apetisser, bornant tout son désir à vivre jusqu'à la relève ».

\section{Le jour et la nuit : tensions symboliques}

Mais au-delà de l'ambiance qu'elle imprime aux journées interminables d'attente, la lumière de l'aube devient, dans certains cas, l'annonce du recommencement de l'horreur, visible. Déjà, dans Gaspard, René Benjamin l'utilisait dans l'avantdernier chapitre pour signifier les changements de sentiments de Gaspard à l'égard de la guerre. En effet, au moment où il se réveille juste avant de s'élancer pour ce qui sera sa dernière offensive (il y perdra une jambe et rentrera donc chez lui), l'aube ne lui livre plus aucune illusion :

Le petit jour qui revient sur ces grelottements, c'est l'heure bla-

farde plus sinistre que les nuits. À cet instant-là, on ne s'étonne pas de mourir; le voile de la mort vous frôle les yeux. L'estomac vide et la bouche tremblante, on reçoit l'ordre de se tenir prêt à l'attaque et de mettre baïonnette au canon. Le cliquetis des armes donne froid. Elles brillent dans l'air blême (Benjamin, $1998: 232$ ).

Seule la lueur des armes brille encore, l'espoir de survivre à l'offensive n'est plus apporté par cet "air blême ». Le paradigme s'est renversé au fil du roman et de l'apprentissage du héros, qui ne sait plus ce qui est le pire, du jour ou de la nuit. Selon les récits, le symbolisme de la nuit se teinte en effet de plusieurs nuances.

"Sinistre » chez Benjamin, nous la retrouvons chez Dorgelès (2013 : 23), tour à tour menaçante, lors de la première nuit des nouveaux camarades, elle est " aux aguets " et "écout[e] la tranchée »; puis révélatrice du destin futur des soldats. Comme ici lorsque le narrateur regarde longuement dormir ses camarades, qu'il distingue à peine dans la nuit, après avoir reçu l'annonce qu'ils devraient attaquer dans deux jours :

Leurs respirations se confondent [...]. Puis il me semble que je ne les entends plus, qu'elles se perdent aussi dans le noir. 
Comme s'ils étaient morts... [...] Ces visages détendus ou crispés, ces faces couleur de terre, j'ai vu les pareils, autour des tranchées, et les corps ont la même pose, qui dorment éternellement dans les champs nus (Dorgelès, 2013 : 74).

Aux "champs muets" font écho les "champs nus " qui évoquent, de nouveau, l'indifférence et la dureté de la réalité de la guerre. La nuit, qui est également le moment où les soldats exécutent les travaux nocturnes de terrassement ${ }^{10}$, effectuent les relèves, font les "coups de mains ", ou encore subissent les bombardements, rend plus présentes la menace et la mort, dans la lignée de tout un pan de son symbolisme traditionnel qui l'associe à la nuit éternelle de la mort. La seule lumière qui lui fasse barrage est celle du foyer provisoire que les camarades ont trouvé dans une ferme. Ils y reviennent périodiquement, pendant environ " deux mois » (Dorgelès, 2013: 93), alternant les jours sur le front et les séjours de repos dans cet endroit qui leur devient familier. Un soir, à l'abri du moulin, les soldats oublient presque leur condition lors d'un "vrai dîner de famille» (Dorgelès, 2013 : 98). Le narrateur ressent un sentiment de sécurité rare :

Il suffit de tirer les gros rideaux et d'allumer la lampe pour se sentir chez soi et ne plus rien craindre. Par prudence, on met encore une toile de tente devant la fenêtre. La nuit n'aura rien de notre chaleur, pas un fil de notre lumière. [...] Mieux que par tous les parapets on se sent défendu par cette lumière qui vous semble si belle après la lueur jaune et dansante des bougies, on se sent défendu par le feu qui ronfle [...] (Dorgelès, 2013 : 97-98).

Lors de cette éclipse de la guerre derrière les rideaux, c'est une nuit tout aussi menaçante que le jour que nous retrouvons.

Dans le dernier chapitre du Feu, Barbusse utilise l'aube comme un révélateur des conséquences du cataclysme qui s'est abattu sur le front, la nuit antérieure : " À demi assoupis, à demi dormants, ouvrant parfois les yeux pour les refermer, paralysés, rompus et froids, - nous assistons à l'incroyable recommencement de la lumière " (Barbusse, 1965 : 405). Paradis et lui peuvent alors mieux comprendre ce que le déluge de la nuit a provoqué chez les hommes : ils sont "presque changés en choses ", comparés à " des escargots " ou encore à une " une rangée immobile de masses grossières, de paquets placés côte à côte »(Barbusse, 1965 : 406). Les deux personnages ne se reconnaissent même pas, malgré leur familiarité, puis ils se montrent incapables de distinguer les soldats allemands des français (Barbusse, 1965 : 408). Pour mieux redoubler le motif de la prise de conscience, après cette vision dantesque, Barbusse

${ }^{10}$ Il est question des «nuits dans la boue " que doivent endurer les soldats dans le chapitre " NotreDame des Biffins » (Dorgelès, 2013 : 138). 
fait se rendormir le narrateur puis il l'éveille de nouveau, en plein " cauchemar » cette fois : "On se réveille. On se regarde, Paradis et moi, et on se souvient. On rentre dans la vie et dans la clarté du jour comme dans un cauchemar " (Barbusse, 1965 : 412). Pour finir, il utilise le thème du monde inversé pour bien insister sur l'incompréhension qui peut naître de la contemplation d'un paysage totalement englouti par le violent orage, d'autant plus qu'un nouvel assaut des intempéries menace : "Les autres, un à un, se dressent. L'orage s'épaissit et descend sur l'étendue des champs écorchés et martyrisés. Le jour est plein de nuit »(Barbusse, 1965 : 434).

L'opposition fréquente entre le jour qui expose à l'ennemi, où la mort est omniprésente, et la nuit est dépassée ici. En effet, dans certains cas, que nous n'avons pas encore évoqués, celle-ci est certes "sinistre" mais peut-être plus favorable à l'oubli de sa condition, comme dans ce passage : "Cependant les heures s'écoulent, et le soir commence à griser le ciel et à noircir les choses; il vient se mêler à la destinée aveugle, en même temps qu'à l'âme obscure et ignorante de la multitude qui est là, ensevelie " (Barbusse, 1965 : 73). Le rapprochement entre le soir et son obscurité et la "destinée aveugle " pointe du doigt le caractère arbitraire de la destinée des soldats, mais il semble également favoriser un oubli relatif, rendant plus cruelle encore la lucidité qu'amène parfois l'aube :

La lumière du jour a fini par s'infiltrer dans les crevasses sans fin qui sillonnent cette région de la terre; elle affleure aux seuils de nos trous. Lumière triste du Nord, ciel étroit et vaseux, lui aussi chargé, dirait-on, d'une fumée et d'une odeur d'usine. Dans cet éclairement blême, les mises hétéroclites des habitants des bas-fonds apparaissent à cru, dans la pauvreté immense et désespérée qui les créa (Barbusse, 1965 : 36).

Synonyme de prise de conscience quotidienne, après le répit relatif de la nuit, la lumière du jour projette sur la guerre un éclairage qui ne permet plus d'enjoliver la réalité, tout apparaît "à cru " et n'a plus rien à voir avec l'imagerie glorieuse et ensoleillée que véhiculent, à travers la presse, les autorités et la propagande guerrière. Comme le montre bien la réaction de Lambert, dans Les Croix de bois, qui s'emporte contre un camarade qui vient de dire, simplement, en contemplant le beau temps, que ce n'est pas un temps à se battre :

- Un temps pour se battre! s'emporta-t-il. C'est dans le PêleMêle que tu as lu ça!... Ah! ils connaissent de bonnes blagues tous les petits coquins qui écrivent sur la guerre... Mourir au soleil, tu parles d'une affaire !... Tiens, je voudrais bien en voir un crever la gueule ouverte dans le barbelé, pour lui demander d'apprécier le paysage... (Dorgelès, $2013: 145$ ).

"Mourir au soleil » n'est plus synonyme de mort glorieuse, mais simplement de mort, dans son sens littéral. Nous trouvons un équivalent à ce passage dans le der- 
nier chapitre du Feu dans lequel Barbusse fait parler le narrateur à la place de Paradis dans une sorte de traduction de sa parole elliptique " "C'est ça, la guerre" » : " Il veut dire, et je comprends avec lui : "[...] C'est cela, cette monotonie infinie de misères, interrompue par des drames aigus, c'est cela, et non pas la baïonnette qui étincelle comme de l'argent, ni le chant de coq du clairon au soleil !" "(Barbusse, 1965 : 413). L'insistance à retravailler les images guerrières en utilisant la lumière solaire de la part des auteurs pourrait signaler la puissance symbolique de cet élément auprès des troupes et des populations. Ainsi, dans Gaspard, quand le narrateur analyse ce qui pousse incidemment les hommes à oublier leur individualité pour se fondre dans la masse sans pensée du régiment, utilise-t-il l'image de l'éclat lumineux :

Ce qui fait l'étrange beauté d'un régiment qui part, c'est d'abord l'uniforme, cette première discipline qui éclate aux yeux. Mais sous des képis pareils, la pensée elle aussi s'égalise, et il semble à chacun que c'est le pas de la Fatalité qu'il emboîte, dès qu'on commande: «En avant... marche! »[...] Que deviennent les amours, les intérêts, les peurs, dans cette mise en route générale, où la cadence du corps emporte les idées ? (Benjamin, $1998:$ 40).

Or, c'est toute cette mise en scène qu'interrogent les récits qui nous intéressent. Dans ceux-ci, la portée symbolique des discours établis ne fonctionne plus pour les soldats dès lors qu'ils entrent en contact direct avec la bataille. Il s'agit alors, pour certains écrivains, comme Barbusse, de créer une nouvelle symbolique à partir d'éléments qui agissent de manière puissante sur notre imaginaire.

\section{L'exemple du Feu : de l'aube blafarde à l'aube aux faibles rayons d'espoir}

L'utilisation de la lumière chez Barbusse nous semble avoir pour fonction de représenter les fluctuations de l'espoir chez les soldats dans cette guerre dont l'issue est incertaine. Nous venons de montrer que la lumière de l'aube devenait synonyme de recommencement, au mieux de l'ennui, au pire de l'horreur et qu'elle fonctionnait alors comme la métaphore de l'éclairage réaliste que porte son récit sur les tranchées.

Toutefois, selon l'endroit où les soldats la voient naître, l'aube ne perd pas totalement son pouvoir de rassurer ou d'étonner les hommes, surtout quand ils s'éloignent des premières lignes et semblent se rapprocher du temps d'avant la guerre, en prenant physiquement leurs distances avec elle. Après une longue marche nocturne, qui doit les mener vers un nouveau cantonnement, derrière les lignes, voici la perception qu'ont les personnages de Barbusse du lever du jour :

Après plusieurs haltes où on se laisse tomber sur son sac, au pied des faisceaux - qu'on forme, au coup de sifflet, avec une hâte fiévreuse et une lenteur désespérante à cause de l'aveuglement, dans l'atmosphère d'encre - l'aube s'indique, se délaie, s'empare de l'espace. Les murs de l'ombre, confusément, 
croulent. Une fois de plus nous subissons le grandiose spectacle de l'ouverture du jour sur la horde éternellement errante que nous sommes (Barbusse, 1965 : 94).

Une nouvelle fois dans le récit, l'aube jette sa lumière crue sur la réalité des hommes et permet de les identifier : pour le narrateur, qui s'inclut dans ce groupe, ils sont devenus une " horde éternellement errante ", tels des barbares. Cette lucidité leur est imposée par le contraste entre la grandeur du spectacle naturel et le désordre et la violence associés au terme " horde ». C'est pourtant ce caractère "grandiose " qui agit sur les soldats, au-delà de la trivialité et des prophéties de "malheur " de certains (Barbusse, 1965 : 95). Ils espèrent alors, de nouveau : "au sortir de la fièvre tumultueuse de la nuit, il semble à tous que c'est d'une espèce de terre promise qu'on s'approche à mesure qu'on marche du côté de l'orient, dans l'air glacé, vers le nouveau village que va apporter la lumière " (Barbusse, 1965 : 95). Très vite des tensions sont à l'œuvre dans cet avènement de la lumière. La marche ne s'arrête pas au premier village traversé et l'apparition du soleil devient un calvaire pour les soldats. L'image "de la clairière féerique qui s'ouvre au milieu des nuages terrestres" (Barbusse, 1965 : 96) s'estompe vite pour faire place à une "route [...] aveuglante ", où il fait trop chaud et qui semble éloigner les soldats de la réalité du cantonnement de par sa longueur.

Même s'ils finissent par y arriver, les soldats, qui nourrissaient l'espoir d'une amélioration dans leur confort par rapport aux cantonnements antérieurs, voient leurs chimères achopper rapidement sur la réalité (Barbusse, 1965 : 99). Ce qui ne les empêche pas, lors de ce répit, de trouver un réconfort ponctuel dans un rayon de soleil : "Parce que le soleil s'est montré, parce qu'on a senti un rayon et un semblant de confort, le passé de souffrance n'existe plus, et l'avenir terrible n'existe pas non plus... “On est bien maintenant." Tout est fini » (Barbusse, 1965 : 122). Éloignés du front, les soldats oublient l'expérience de la guerre. Mais nous pouvons aussi interpréter ce "tout est fini" comme le soulagement éphémère de la bête de somme qui donne une autre nuance à la guerre : elle semble totalement étrangère à la destinée humaine.

De fait, la lumière et son renouveau quotidien, dans sa grandeur et sa temporalité propre sont aussi étrangers aux intérêts particuliers des hommes que l'est la guerre. C'est ce dont fait l'expérience Lamuse lors des heures de liberté que lui octroie ce premier jour au cantonnement. "Dans la clarté impitoyable de l'éternel printemps " (Barbusse, 1965: 119), il tente d'arracher de force un baiser à Eudoxie, une jeune femme qui suit l'escouade car elle entretient une relation avec un des camarades de Lamuse. L'excès de lumière et de chaleur - «La lumière éclate partout, chauffe et cuit le creux du chemin, y étale d'aveuglantes et brûlantes blancheurs çà et là, et vibre dans le ciel parfaitement bleu" (Barbusse, 1965: 117) -, semblent avoir une influence sur la scène. Il augmente la séduction de la jeune femme à la «blancheur lu- 
naire ", "tentante dans le soleil » (Barbusse, 1965: 118) aux yeux de Lamuse. De plus, il renforce l'aveuglement du soldat qui s'est totalement mépris sur les raisons de la présence d'Eudoxie au cantonnement. Incapable de contrôler ses désirs, il s'obstine, malgré la répugnance évidente qu'il inspire à la jeune femme : "Il mourrait pour la toucher avec sa bouche» (Barbusse, 1965 : 118). Elle arrive toutefois à se dégager rendant inutile l'intervention - tardive - du narrateur, car Lamuse revient, avec ce geste, à la raison.

Le déroulement de la scène conduit à une représentation pathétique de Lamuse - non exempte d'une victimisation certaine du personnage masculin de la part $\mathrm{du}$ narrateur, qui rend la lecture de cette scène difficile aujourd'hui ${ }^{11}$. La violence qu'il exerce sur la jeune fille est fortement atténuée par celle exercée par le printemps et les désirs qu'il fait naître chez cet homme, dans un contexte où les femmes sont absentes de la vie quotidienne des soldats. Il nous semble alors que le rapprochement de la guerre et des cycles naturels, à travers la notation de la "clarté impitoyable " de la saison et de la comparaison de ce moment avec le "commencement des âges " (Barbusse, 1965 : 119), sert ici à esquisser l'image du soldat en martyr que Barbusse élabore tout au long de son récit. Comme pour mieux assimiler l'indifférence de la nature et de la guerre et rendre de nouveau possible un discours d'espoir. Si l'homme ne capitule pas face au cycle des saisons et aux retours périodiques des cataclysmes, pourquoi ne pas adopter la même attitude face à la guerre ?

Pour accentuer ce rapprochement entre deux forces aveugles, deux personnages sont emblématiquement placés sous le signe de la vitalité et de la lumière pour « mieux » être anéantis par la guerre. Le premier personnage est Eudoxie elle-même : la jeune femme était apparue pour la première fois aux soldats à l'orée d'un bois dans un rayon de soleil telle une biche (Barbusse, 1965 : 89). Dans l'épisode avec Lamuse, ses cheveux "d'une intense blondeur" ressemblaient à des "flammes" (Barbusse, 1965 : 118) et elle dégageait " la force lumineuse d'une enfant ». Ce sont ces cheveux que Lamuse reconnaît - de manière encore bien symbolique - quand il découvre son cadavre en creusant une sape (Barbusse, 1965 : 256-257). La mort de celle qui représente une incursion de l'amour et de la sensualité sur le front, permet à Barbusse d'utiliser une nouvelle fois l'image de l'anéantissement aveugle des forces vitales par la guerre.

Dans nombre de récits prenant pour sujet la Grande Guerre, les comparants naturels servent aux auteurs à assimiler les déchaînements du front à un cataclysme naturel. Rapidement considérée comme moderne, la guerre des machines rappelle

${ }^{11}$ Nicolas Beaupré (2003 : 87) montre que, pendant le conflit, les institutions de la censure ont renforcé l'image de victimisation des soldats véhiculée par les récits de témoignages : les passages évoquant l'animalisation, la brutalisation des soldats, les moments de violence guerrière assumés étaient, eux, plus susceptibles d'être supprimés. Il remarque le même mécanisme de gommage des "moments les plus atroces de la violence infligée » dans les récits littéraires des années 20. 
toutefois l'impuissance de l'homme face aux catastrophes naturelles : le titre du roman de Jünger, Orages d'acier (1920) est, à ce titre, significatif, ainsi que celui du récit de Barbusse lui-même, qui emploie la métaphore, attestée dès le XIX siècle, du phénomène naturel de l'incendie pour désigner le combat. Barbusse ne se contente pas de cette métaphore et va plus loin dans ce passage où Porteloo retrouve son village complètement anéanti : "À mesure qu'on avance, tout apparaît retourné, terrifiant, plein de pourriture, et sent le cataclysme" (Barbusse, 1965 : 199).

Le même mécanisme qu'avec Eudoxie est à l'œuvre avec la destinée de Poterloo. La différence se situe dans la portée et la fin de l'épisode qui oriente la lecture vers le sens sacrificiel de l'épreuve du soldat. Un matin, à la faveur du brouillard qui permet d'éviter d'être repéré par les Allemands (qui continuent à bombarder régulièrement le village perdu), Poterloo réussit à retourner à Souchez, dont il est originaire, avec le narrateur. Il éprouve des difficultés à retrouver sa maison. Aucun point de repère ne lui permet de la situer, sauf une pierre qu'il repère in extremis, juste avant que le brouillard ne commence à se lever et les force à rebrousser chemin. Brisé par la vision qu'il vient d'avoir : "- Tu vois, c'est trop, tout ça. C'est trop effacé, toute ma vie jusqu'ici. J'ai peur, tellement c'est effacé " (Barbusse, 1965 : 205) ; il trouve pourtant des ressources dans l'apparition du jour sur Souchez, détruit au loin, et y voit une promesse de renaissance future : "À la faveur de la distance et de la lumière, la petite localité se reconstituait aux yeux, neuve de soleil!»(Barbusse, 1965: 211). Petit à petit, il médite et en arrive à cette conclusion :

"Après tout, c'est la vie, et on est fait pour refaire, pas? On r'fera aussi la vie ensemble et le bonheur; on refera les jours, on refera les nuits.

Et les autres aussi. Ils referont leur monde. Veux-tu que je te dise ? Ça sera peut-être moins long qu'on croit... " (Barbusse, $1965: 213)$.

L'emploi du préfixe « re » martèle le discours de Porteloo qui semble vouloir se convaincre lui-même tout en ne pouvant s'empêcher d'embrasser totalement le symbolisme de l'aube et du renouveau - depuis une position géographique élevée -, avant de redescendre définitivement vers les lignes :

Quand nous arrivâmes en haut de la côte, il se retourna et jeta un dernier coup d'œil sur les lieux massacrés que nous venions de visiter. [...] Mieux encore que tout à l'heure, le beau temps disposait sur ce groupement blanc et rose de matériaux une apparence de vie et même un semblant de pensée. Les pierres subissaient la transfiguration du renouveau. La beauté des rayons annonçait ce qui serait, et montrait l'avenir. La figure du soldat qui contemplait cela s'éclairait aussi d'un reflet de résurrection. Le printemps et l'espoir y déteignaient en sourire; et ses joues 
roses, ses yeux bleus si clairs et ses sourcils jaune d'or avaient l'air peints de frais (Barbusse, 1965 : 214-215).

Poterloo est transfiguré, il incarne ici une figure christique grâce au " reflet de résurrection » qui colore son visage. Son espoir, né du renouveau du jour, s'est propagé chez tous les soldats, y compris le narrateur, qui ont ressenti le même effet du « soleil » sur les tranchées:

Nous avons eu de mauvais jours, des nuits tragiques, dans le froid, dans l'eau et la boue. Maintenant, bien que ce soit encore l'hiver, une première belle matinée nous apprend et nous convainc qu'il va y avoir bientôt, encore une fois, le printemps. $[\ldots] \mathrm{Oh}$ ! La guerre va finir, on va revoir à jamais les siens [...] et on leur sourit dans cet éclat jeune qui, déjà, nous réunit (Barbusse, 1965 : 215-216).

Après ce passage plein d'espoir, la progression des deux soldats dans leurs lignes, côte à côte, se poursuit. Mais le temps change soudainement. Le narrateur et Poterloo arrivent à leur tranchée juste à temps pour constater qu'il va pleuvoir pour la relève, qui les éloignera une fois de plus du front et de la menace de la mort. Ils se mettent en route sous une pluie battante, Poterloo "avec sa puissante carrure ", son " dos fort, solide comme une colonne d'arbre, comme la santé et l'espoir " (Barbusse, 1965 : 220); et le narrateur, convaincu que ce revirement météorologique n'affectera pas son camarade, revenu des ruines de son foyer avec une force décuplée : "Ce n'est pas une averse qui lui ôtera le contentement qu'il emporte dans son cœur ferme et solide, et ce n'est pas une maussade soirée qui éteindra le soleil que j'ai vu, il y a quelques heures, entrer dans sa pensée " (Barbusse, 1965 : 217). Comme pour éloigner ce qui pourrait paraître un mauvais présage, le narrateur ne cesse de commenter la transformation de Poterloo, une transformation que les aléas du temps ne pourraient amoindrir. Toutefois, juste avant la fin du trajet, Barbusse achève de construire son contraste en faisant atteindre au chapitre le summum du pathétique, dans sa dernière phrase.

En effet, un obus fait voler Poterloo dans un " éclair de tonnerre » et le narrateur, encore sous le coup de l'étourdissement de l'explosion, de témoigner : "j'ai vu son corps monter, debout, noir, les deux bras étendus de toute leur envergure, et une flamme à la place de la tête " (Barbusse, 1965 : 220). Le parallèle christique s'achève logiquement sur la mort du personnage, dont l'aspect spectaculaire dépasse clairement la notation réaliste pour atteindre au symbole : grâce à l'ascension du corps et à l'évocation des bras qu'on devine dessiner la posture du Christ sur la Croix ${ }^{12}$.

${ }^{12}$ Au sujet de cette mort et de l'écriture de Barbusse, Jean Norton Cru écrit : "Barbusse se souvient plutôt de la Bible illustrée que des faits réels du front " (Témoins. Essais d'analyse et de critique des souvenirs de combattants édités en France de 1915 à 1928, Presses Universitaires de Nancy, 1993 [réédition de l'édition de 1929], p. 559). Cité par Esther Pinon (2015: 859). 
L'apparente facilité du symbolisme et du contraste masquent mal le besoin de redonner un sens à un conflit qui l'a perdu sous l'action répétée de l'horreur. Ainsi, Esther Pinon interprète-t-elle la mort de Poterloo comme entrant dans cette représentation du recommencement incessant de l'horreur sur le front : "Les combattants tués sont des Christs de l'échec, qui disent l'éternel recommencement de l'horreur et l'impuissance de la fraternité " (2015: 859). Nous suivons cette ligne, en ajoutant que le texte n'est pas pour autant exempt d'ambigüité à cet endroit. En effet, elle nous semble provenir du fait que l'image de la destruction prépare celle du recommencement.

L'auteur du Feu exploite au maximum les notations de lumière et leurs variations dans des nuances qui traduisent parfaitement les hésitations d'un texte qui dénonce la guerre tout en tentant de lui conférer un sens ultime. Ce qu'explique Nicolas Beaupré quand il signale l'ambigüité des récits adoptant la "posture compassionnelle " qui, certes, montrent la souffrance des soldats, grâce au registre pathétique, mais ne "remettent pas fondamentalement en cause la guerre elle-même " (2006 : 146). Dans le dernier chapitre du Feu, alors que la fin de la guerre n'est toujours pas en vue, puisque le récit est publié en plein conflit, Barbusse fait naître une "aube d'espoir " sur le paysage de fin du monde et de déluge dans la dernière phrase du récit :

Et tandis que nous nous apprêtons à rejoindre les autres, pour recommencer la guerre, le ciel noir, bouché d'orage, s'ouvre doucement au-dessus de nos têtes. Entre deux masses de nuées ténébreuses, un éclair tranquille en sort, et cette ligne de lumière, si resserrée, si endeuillée, si pauvre, qu'elle a l'air pensante, apporte tout de même la preuve que le soleil existe (Barbusse, 1965 : 435).

L'idée du " recommencement » fait écho à la disparition de Poterloo ainsi que cette assertion, "Le soleil existe ", qui sert de conclusion à un texte qui insère les effets de lumière dans une représentation cyclique du temps. Ce qui autorise à considérer les sacrifices consentis comme une nécessité pour pouvoir parler de renaissance ou de recommencement. Celui-ci n'est pas toujours accordé à ceux qui sont en première ligne, même s'ils croient en sa possibilité, envers et contre tout. Tout comme la nature, la guerre est aveugle. Barbusse propose alors une manière de l'affronter qui utilise tous les ressorts de la symbolique de l'aube nouvelle après l'épreuve et écrit un texte " qui annonce métaphoriquement un ordre nouveau à bâtir (la thématique de l'Aube (chap. XXIV) et de la Clarté si obstinément déclinée par Barbusse) " (Pudal, 2015 : 787). S'il est vrai qu'il contribue à lancer un type de récit (Compagnon, 2004 : 17), tous les écrivains ne donnent pas à leur texte ce halo d'espoir. 


\section{Ombres au tableau}

Dans les deux textes publiés à la fin du conflit, en 1919, Les Croix de bois et Clavel soldat, les auteurs portent un regard plus désabusé sur la guerre et ses conséquences. Contrairement à Barbusse, Dorgelès, utilise la symbolique de l'obscurité pour clore son texte. Pour Werth, les effets de lumières permettent de mettre à distance très rapidement l'acte de représentation de la guerre et de relativiser ses significations.

Dans le dernier chapitre des Croix de bois, le narrateur dresse un bilan rétrospectif de son expérience, à la lueur retrouvée de « la lampe tranquille » du foyer (Dorgelès, 2013 : 244), tout en tentant de regarder vers l'avenir : selon lui, l'oubli est inévitable et il ensevelira une deuxième fois les soldats morts aux combats. Une seconde mort, symbolisée par la "nuit éternelle ", va s'étendre sur eux et prolonger leur martyre (Dorgelès, 2013 : 245). Pour lui, les morts seront exclus de la renaissance car elle ne les verra pas revenir et participer du mouvement général de reconstruction. D’un moment de la guerre à l'autre, les écrivains opposent à l'éclat d'une gloire promise sur le champ d'honneur une aube qui transfigurerait le sacrifice des uns pour éclairer le recommencement des autres; ou une "nuit éternelle " qui anéantira définitivement les promesses de survie des sacrifiés au-delà du conflit.

Léon Werth qui a écrit son texte entre 1916 et 1917 mais ne peut le publier qu'en 1919, va plus loin encore et ouvre directement Clavel Soldat par une réflexion sur ce qu'évoque le paysage chez le personnage principal dont on ne sait encore rien :

C'est un contraste de chromo, une petite rêverie bien sage sur la guerre, qui va commencer : des vaches montent la route au crépuscule, d'une marche dodelinante. [...] Elles vont à l'abreuvoir en contrebas, sous les arbres, sous de vieux arbres, à l'abreuvoir de vieilles pierres. Le soir et l'ombre semblent une image pacifique. André Clavel n'a pas vu cette image pour ellemême. Elle s'oppose à d'autres images, qui sont en lui, très vagues, et très fermement attachés : les images de la guerre, le tumulte des batailles (Werth, $2006: 7$ ).

La notation de l'orage, pour signifier le début de la guerre de Benjamin et de Barbusse se mue en réflexion ironique. Dans ce premier paragraphe du texte, le lecteur est d'emblée placé devant le fait que le paysage et sa lumière fournissent un support idéal au symbolisme: grâce aux contrastes qu'ils autorisent, à leur pouvoir d'expression mais aussi à leur caractère stéréotypé, qui en facilite la circulation ( chromo »). Cette image, que Clavel " ne voit pas pour elle-même " mais parce qu'elle "s'oppose à d'autres images", nous montre que l'écrivain introduit son lecteur dans un texte qui met à distance ses effets. Dès le seuil, Werth met en perspective l'acte de figuration et d'interprétation des images de la guerre, notamment en insistant sur les effets de lumières qui constituent, pour les personnages et, par extension, 
pour les lecteurs, des indices ou des signes qui rappellent d'autres images et entrent en résonnance avec elles.

À la fin du texte, comme en réponse à la fin proposée par Barbusse à son récit, la présence d'un rayon de soleil ne fait pas espérer Clavel. Alors qu'il s'éloigne des premières lignes, pour porter un message, et qu'il pourrait ainsi bénéficier de l'éloignement du front dont nous avons parlé dans le récit de Barbusse, Clavel regarde la campagne environnante :

Entre les nuages passe un peu de soleil. [...] Si ce n'était la guerre, comme il accueillerait ce paysage! Il tente de retrouver cette bienveillance à la nature du citadin à la campagne. Il y aurait là la maison du paysan louée pour l'été. La fille de quinze ans conduirait ses vaches. Il poserait ses yeux sur les champs et la plaine, comme s'ils allaient être lavés d'une longue fatigue. Malgré ce triste ciel lorrain, malgré ce ciel dévot, il sentirait alors que la vie peut être neuve. Mais la campagne même se lie pour lui à la guerre, est teintée de guerre (Werth, 2006 : 359).

Contrairement à la vision inaugurale, tout contraste entre la nature paisible, "pacifique ", et la guerre est aboli. Le conditionnel marque l'impossibilité pour le personnage d'oublier la guerre. Il ne peut renouer avec son ancienne vision de la nature, tout aussi artificielle - comme on le voit avec l'emploi du terme appartenant au domaine de la peinture "paysage " et avec l'aveu de l'idéalisation de la campagne par le citadin -, que les visions de la guerre qui lui sont venues à l'esprit dans l'incipit. Contrairement au texte de Barbusse, le lien entre nature et guerre ne provoque aucune image de renouveau. Il implique seulement que certains se représentent ces deux réalités sur un même plan pour mieux espérer, alors que d'autres n'en sont plus capables.

Dans ce récit, le lexique de la lumière est alors associé à plusieurs reprises à celui de la représentation ("chromo, image») par le biais, notamment, de celui de la peinture (" paysage, teintée »), car ce qui est en jeu pour Clavel, c’est le constant besoin de se représenter la guerre sous un jour ou un autre :

La réalité profonde de cette guerre n'est pas la tranchée, n'est pas l'attaque. Elle est dans la façon dont chacun colore son idée de la guerre selon la tranchée, selon l'attaque, l'embuscade dans le régiment ou à l'arrière, le grade. (Werth, 2006 : 353-354).

Depuis la perspective de Clavel, la lumière qui « colore » la guerre s'assombrit tout au long du texte :

Le petit jour aux tons cadavériques fait un paysage de réveil en chemin de fer. 
Sous un ciel de nuages noirs, sous un ciel sans nuances, un ciel de manuel de météorologie, Clavel joue aux dames avec le curé Évariste (Werth, 2006 : 358-359 et 360-361).

Avec ce rythme ternaire qui raille le lyrisme de la guerre et son tragique, ainsi que cette image aux accents surréalistes avant la lettre, Léon Werth ne cesse de remettre sur le métier une description qui puisse exprimer ce que son personnage tente de faire, chaque matin, en se réveillant, que ce soit sur le front ou en cantonnement, sous l'uniforme : comprendre. Comme l'exprime Georges Duhamel quand il mêle le motif du réveil au "petit jour " à celui de la quête du sens dans un environnement opaque : "Levé avec le petit jour, je voguais à travers le brouillard et cherchais à comprendre » (Duhamel, $1967: 25)$.

\section{Conclusions}

Le travail de ces quatre écrivains sur les représentations de la guerre, dans toutes les tensions qu'il fait affleurer sur le sens individuel et collectif d'une telle expérience, nous semble particulièrement visible dans l'utilisation du paysage, hautement signifiant dans notre tradition littéraire, et de la lumière qui lui est liée. Dans ces deux versants, artificiel et naturel, l'éclairage sert à signifier l'impression d'extrême étrangeté. Il jette des lueurs fantastiques sur le texte, arrivant parfois, par une sorte d'excès de réalisme, à la fantaisie la plus étrange, pour mieux figurer la difficulté à représenter un espace du front totalement étranger au monde passé et au monde de l'arrière.

D'autre part, parmi les éclairages choisis par les écrivains, la lumière symbolise tour à tour le faux éclat de gloire des discours officiels sur la guerre; le regard réaliste porté sur le conflit; l'ennui attaché à l'expérience et le manque de perspectives, d'horizon d'attente, quand elle est tamisée par les brumes ; quand elle éclaire de nouveau le champ de bataille, l'horreur répétée ; et parfois les fluctuations du sentiment d'espoir chez les soldats. La communauté d'expérience s'instaure alors sur le partage d'un même sentiment, ressenti lors des mêmes aubes tristes et désolées, comme le suggère le narrateur de "Sur la Somme» de Duhamel :

Le matin vint. Ceux qui auront vu les aubes de la guerre, après les nuits employées à combattre ou consumées dans la sanglante besogne des ambulances, ceux-là connaîtront une des plus grandes laideurs et une des plus grandes tristesses du monde.

Pour ma part, je n'oublierai jamais cette lumière avare et verte, cet aspect découragé des lampes et des visages, cette odeur suffocante des hommes envahis par la pourriture, ce frisson du froid matinal, pareil au dernier souffle glacé de la nuit dans les frondaisons engourdies de grands arbres (Duhamel, 1967 : 32).

Les aubes qu'ont connu les combattants deviennent une nouvelle image consacrée, qui tente d'atteindre des lecteurs qui ne les ont pas forcément connues; et 
témoigne d'une expérience individuelle et surtout collective. Cette image met également en doute la faculté des humains à se souvenir de tels événements : qu'en resterat-il après la mort des derniers survivants qui, eux, ne pourront l'oublier ? Leur expression littéraire, à n'en pas douter.

\section{RÉFÉRENCES BIBLIOGRAPHIQUES}

BARbusSe, Henri (1965 [1916]) : Le Feu. Paris, Le livre de Poche.

BENJAMIN, René (1998 [1915]) : Gaspard. Paris, L'Archipel.

BEAUPRÉ, Nicolas (2006) : Écrire en guerre, écrire la guerre France, Allemagne 1914-1920. Paris, CRNS Histoire.

BEAUPRÉ, Nicolas (2013) : «La guerre comme expérience du temps et le temps comme expérience de guerre: Hypothèses pour une histoire du rapport au temps des soldats français de la Grande Guerre ». Vingtième Siècle. Revue d'histoire, 117/1, 166-181. DOI : $10.3917 /$ vin.117.0166.

CAMPA, Laurence (2010) : Poètes de la Grande Guerre, Expérience combattante et activité poétique. Paris, Classiques Garnier.

COLETTE (1984) : Les Heures longues, in Euvres, t. II. Paris, Gallimard, 475-580.

DORGELÈs, Roland (2013 [1919]) : Les Croix de bois in 1914-1918 Français et Allemands dans les tranchées. Paris, Le livre de poche.

DuHAMEL, Georges (1967 [1918]) : Civilisation. Paris, Le Livre de Poche.

FOUCHARD, Flavie (2017) : "Échos de la Grande Guerre. La prolifération des voix dans Gaspard, Le Feu, Les Croix de bois et Clavel Soldat ", in Nicolas Bianchi et Toby Garfitt (éd.), Writing the Great War / Comment écrire la Grande Guerre ?. Oxford, Peter Lang, 139-160.

KAEMPFER, Jean (1998) : Poétique du récit de guerre. Paris, José Corti, 1998.

LEDDA, Sylvain (2015) : "Requiem ou symphonie funèbre ? Notes sur la mort dans Le Feu ». Revue d'histoire littéraire de la France, vol. 115(4), 825-838. DOI:10.3917/rhlf.154.0825.

PINON, Esther (2015) : "Du Feu à Jésus : Henri Barbusse et le sacré ». Revue d'histoire littéraire de la France, 115/4, 855-868. DOI:10.3917/rhlf.154.0855.

PUDAL, Bernard (2015) : «Barbusse : jalons pour une étude d'un capital symbolique singulier ". Revue d'histoire littéraire de la France, 115/4, 783-796. DOI : http://10.3917/rhlf.154.0783.

SCHOEnTJes, Pierre (2009) : Fictions de la Grande guerre, Variations littéraires sur 14-18. Paris, Classiques Garnier.

SCHOENTJES, Pierre (2011) : "Images de la nature dans les romans de la Grande Guerre : esquisse d'une typologie ». Études littéraires, 42/2, 123-138. 
TOURET, Michèle (2001) : «La guerre 1914-1918 est-elle une période littéraire ou un facteur de périodisation ?", in Francine Dugast-Porte et Michèle Touret (dir.), Le Temps des lettres: Quelles périodicisations pour l'histoire de la littérature française du 20e siècle ? Rennes, PUR, 211-221.

WERTH, Léon (2006 [1919]) : Clavel Soldat. Paris, Viviane Hamy. 